\title{
DÜBLIN
}

Technological University Dublin ARROW@TU Dublin

2006-01-01

\section{A wavelet controller for shunt active power filter}

\author{
Malabika Basu \\ Technological University Dublin, mbasu@tudublin.ie \\ Biswajit Basu \\ Trinity College Dublin
}

Follow this and additional works at: https://arrow.tudublin.ie/engscheleart

Part of the Electrical and Computer Engineering Commons

\section{Recommended Citation}

Basu, Malabika and Basu, Biswajit :A wavelet controller for shunt active power filter. 3rd IET International Conference on Power Electronics, Machines and Drives, Dublin, Ireland, 2006, pp.76-79. doi:10.21427/ 378t-qk56

This Conference Paper is brought to you for free and open access by the School of Electrical and Electronic Engineering at ARROW@TU Dublin. It has been accepted for inclusion in Conference papers by an authorized administrator of ARROW@TU Dublin. For more information, please contact arrow.admin@tudublin.ie, aisling.coyne@tudublin.ie,gerard.connolly@tudublin.ie.

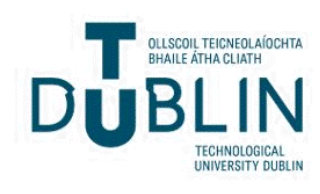




\title{
A Wavelet Controller for Shunt Active Power Filter
}

\author{
Malabika Basu*, Biswajit Basu ${ }^{\dagger}$ \\ *School of Control Systems and Electrical Engineering, Dublin Institute of Technology, Ireland \\ email:mbasu@dit.ie \\ ${ }^{\dagger}$ School of Engineering, Trinity College Dublin, Ireland \\ email: basub@tcd.ie
}

Keywords: active power filter, reference current generation, wavelet analysis

\begin{abstract}
A wavelet-based controller for shunt active power filter (APF) has been proposed in this paper. The constructed controller based on multi resolution analysis has the advantage of better frequency bandwidth selection. The tuning parameters of the controllers are the gains to be applied to the time-frequency signals at different resolutions. This has the advantage of aiding the process of a proper choice of coefficients of tuning parameters. The developed simulation model of the APF with wavelet-based control shows satisfactory steady state and dynamic performance. A comparison with standard PI controller shows that the dynamic response of the proposed wavelet controller is much faster. This is of advantage in specific applications where the fast dynamic response of the controller is of importance to achieve a desired reference within a short duration of time. The novel technique of the generation of control signal ensures that even when the steady state error is zero, the controller can generate a steady control signal. The wavelet data window width is chosen optimally such that frequencies above $600 \mathrm{~Hz}$ are eliminated. This avoids the generation of high frequency spikes in the reference control current signal and ensures stability.
\end{abstract}

\section{Introduction}

Among the controllers available for industrial applications, PID controller is probably the most popular one. There has been several variations of the PID controller available e.g., nonlinear PID with nonlinear gains. The PID controller is easy to implement and tune [1]. The basic PID controller works on the principle of the construction of control gain as a linear combination of the error itself representing the present, the integral of the error representing the past and the derivative of the error representing the future/trend. The controller is tuned with proper gain parameters, which are multiplied with the three previously mentioned components. Based on the frequency content the three components, i.e., the error, the integral of the error and the derivative of the error could be related to the low and high frequency contribution in the error signal. This motivates resolving the signal using wavelet analysis and using a linear combination of the timefrequency signals at different bands to construct a controller.

The combined time-frequency information is found to be very useful in PQ conditioning and monitoring applications [2-4]. The local frequency content information (temporal) could lead to the control of PQ signal aptly in time domain. With this perspective a new controller has been designed for shunt active power filters (APF) in generating the ideal reference current to curb and buffer the harmonic current problems in the utility by proper switching of the inverters. The error of the dc link voltage signal with respect to the reference is resolved through Multi Resolution Analysis (MRA) [5, 6] into several frequency bands of interest. Multiplied by appropriate gain coefficients, the approximate coefficients and the detail coefficients of the frequency bands generate the ideal reference magnitude of the current to be drawn from the supply. The hysteresis controller compares the actual source current with the reference current to trigger the APF switches appropriately and achieve the desired filtering effect.

In the following sections the design of the wavelet controller is discussed. A comparative case study is performed where it is found that the wavelet controller is much faster than the conventional PI controller in case of dynamic response.

\section{Decomposition of the error signal using Multi Resolution Analysis (MRA)}

The idea of Multi Resolution Analysis (MRA) is very similar to sub-band decomposition where a signal is divided into a set of signals each containing a frequency band. In MRA the input at each stage is always split into two bands in time; the higher band becomes one of the outputs, while the lower band again is further split into two bands. This procedure is continued till a desired resolution is achieved [6].

Consider an error sequence e $(n), n=0,1,2, \ldots . n-1$. With a low pass filter and a high pass filter, the MRA scheme decomposes $e(n)$ into its sub-band components of $d_{n}{ }^{1}, d_{n}{ }^{2} \ldots$. , $d_{n}{ }^{L}$, and $c_{n}{ }^{L}$ representing the coarsest, which is the dc component as $\mathrm{L}$ tends to $\propto$. This is mathematically represented as: 


$$
e(n)=c_{n}^{L}+\sum_{i=1}^{L} d_{n}^{i}
$$

The DC link voltage signal has been taken as the feedback signal for the wavelet control.

The digital filters $\mathrm{g}$ and $\mathrm{h}$ determine the wavelet basis function $\psi(t)$ and the associated scaling function $\phi(t)$. These two functions are given by two-scale equations

$$
\phi(t)=2 \sum_{l} \bar{g}(l) \phi(2 t-i)
$$

and

$$
\psi(t)=2 \sum_{l} \bar{h}(l) \phi(2 t-i)
$$

for a dyadic wavelet construction. For perfect reconstruction, the coefficients $\bar{g}$ and $\bar{h}$ respectively must satisfy the relationship

$$
\begin{gathered}
g(l)=\bar{g}(p-1-l) \\
h(l)=\bar{h}(p-1-l)
\end{gathered}
$$

where, the delay $p-1$ is the filter order for the chosen filter, which is related to the wavelet basis function.

The time-frequency signals of (1) are given by

$c_{n}^{L}=\int s(t) \phi_{L n}(t) d t$

$d_{n}^{i}=\int s(t) \psi_{\text {in }}(t) d t$

with

$$
\begin{aligned}
& \phi_{L n}(t)=2^{-\frac{L}{2}} \phi\left(\frac{t}{2^{L}}-n\right) \\
& \psi_{\text {in }}=2^{-\frac{(i-2)}{2}} \sum h(l) \phi\left(\frac{t}{2^{i-1}}-p+1-2_{n}\right)
\end{aligned}
$$

\section{Synthesis of the wavelet controller and the control of APF}

Since, the time-frequency signals in (1) obtained by wavelet analysis provides information about the system and its dynamics at different scales, these signals can be appropriately combined to generate a control to meet desired characteristics. The general form of a control obtained from the linear combination of the time-frequency signals at different scales is given by
$u=K_{L} C_{n}^{L}+\sum_{i=1}^{L} K^{i} d_{n}^{i}+\int \sum_{w}^{L} \bar{K}_{i} d_{n}^{i} d t$

In equation (10), the parameters $K^{i}, \bar{K}_{i}(\mathrm{i}=1,2, \ldots, \mathrm{L})$ and $K_{L}$ are the gain coefficients and are to be chosen to achieve a desired response target and $w$ is the window width of integral control. If the contribution of the energy in any of the bands is not significant, it is directly reflected on the control and hence the bandwidth of the controller is automatically adjusted in this form. In the present paper db4 mother wavelet has been used. The schematic of the control is shown in Fig 1. The error signal (dcVerr(n)) has been decomposed up to 24 levels. The reconstructed signal $\left(a_{24}=c_{24}^{L}\right)$ using the approximate coefficients at this level correspond to frequency lower than $0.0015 \mathrm{~Hz}$, which carries the DC information of the error voltage. This is used to design a part of the controller such that the constant dc link voltage is achieved. In addition, another part of the controller is based on a signal which is the complementary part of the signal $a_{24}$ and is denoted by $\bar{a}_{24}$, where, $\bar{a}_{24}=\left(\sum_{i=1}^{24} d_{n}^{i}\right)$. The two signals $a_{24}$ and $\bar{a}_{24}$ constitute the total error signal e(n). For the second part of the controller, the considered signal $a_{24}$ is integrated over a time window, $w$, to have the control effect even when the instantaneous error tends to zero. The time window has been chosen optimally so that frequencies above a certain limit $(600 \mathrm{~Hz})$ which are beyond the range of interest are eliminated. This also avoids the generation of instantaneous spikes in the reference signal of control current. The total control is a linear combination of two parts with appropriate gains. The gain parameters considered in this paper are

$$
K_{L}=15000 ; K^{i}=0, \bar{K}_{i}=150=\bar{K}, \mathrm{i}=1,2, \ldots 24 .
$$

Finally, the control action is averaged over half the cycle of the fundamental power frequency to reduce the harmonic content of the reference supply current. The final reference current $\left(\mathrm{i}_{\mathrm{s}}{ }_{\mathrm{s}}\right)$ is generated by multiplying $\mathrm{u}$ with the sinusoidal template of the supply voltage.

The developed controller is applied to control the DC link voltage of a shunt active power filter (APF) and generate the desired supply current reference. It is well known that APF is a controlled VSI connected parallel to the load (linear or nonlinear) $[6,7]$. The APF controls its switching in such a way that the overall current demand of the load is decoupled in the following way. The fundamental active component of current is drawn from the utility. The reactive component of current and major components of harmonics (in case of nonlinear loads) are provided by the APF. Thus the utility harmonic current pollution is controlled and the VAR demand from the utility is minimised. Therefore, generating the reference 
current demand is a key part of the effective control of APF. The switching of the APF through hysteresis controller is a fairly standard procedure that has been used in this paper [9].

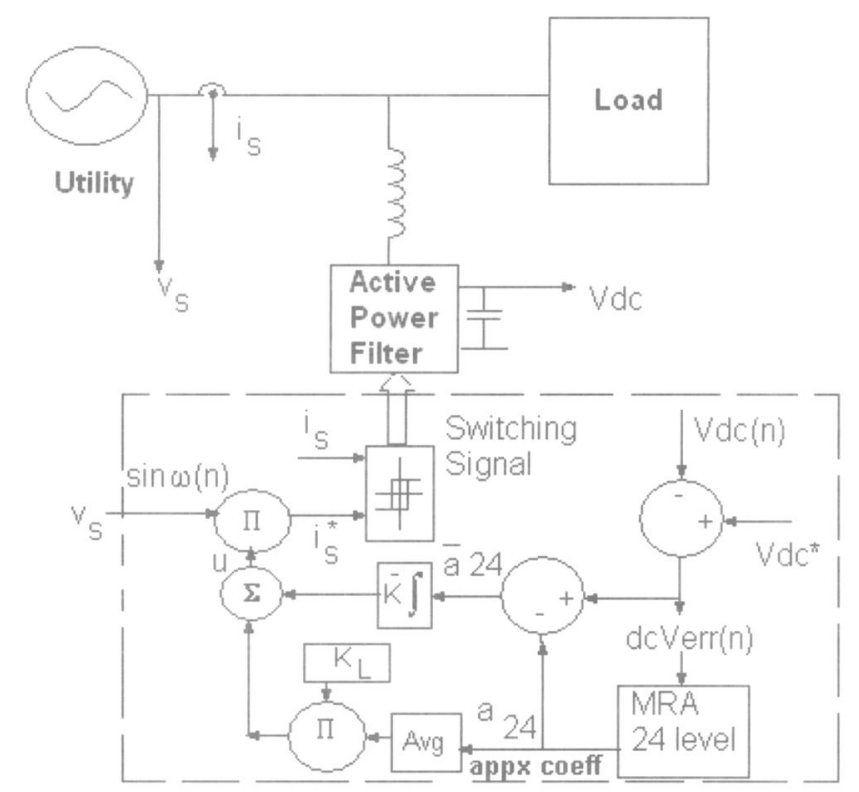

Figure 1 Wavelet controller schematic for the Active Power Filter

\section{Simulation Results}

The simulation of the proposed wavelet based APF is carried out using MATLAB on a $230 \mathrm{~V}$ distribution system on a 5 KVA linear load, with varying power factor. Figure 2 shows the supply voltage and current which are in phase for different load and power factor conditions for a set DC voltage reference of $400 \mathrm{~V}$. The load power factor has been subjected to a dynamic step change from 0.86 (lag) to 0.64 (lag) during the time period of 0.32 to $0.6 \mathrm{sec}$, and restored to 0.86 (lag) after $0.6 \mathrm{sec}$. It is seen that the controller can successfully track the demand of the active component of current. The supply current power factor is maintained at unity. The DC link voltage of the APF corresponding to the load change in Figure 3 is also plotted. The actual DC link voltage is maintained at the desired level $(400 \mathrm{~V})$, and the dynamic response of the dc link controller to load change is very fast. These two typical results show the effectiveness of the APF with the wavelet controller. Similar test simulation has been carried out with a DC link conventional PI controller and similar responses are plotted in Figures 4 and 5. Though the steady state performance from the utility current point of view is similar, during load change there is more oscillation in the supply current as the dc link voltage takes more time to be restored to its desired reference.

In Figure 4 a slow frequency oscillation is initiated in the supply current drawn by the load in response to the load power factor change. The multi resolution based control can provide much faster dynamic voltage response for the DC link voltage compared to the conventional PI controller, hence the variation in load change is faster reflected in the supply current. The better dynamic response is achieved as the wavelet controller is based on local frequency information.

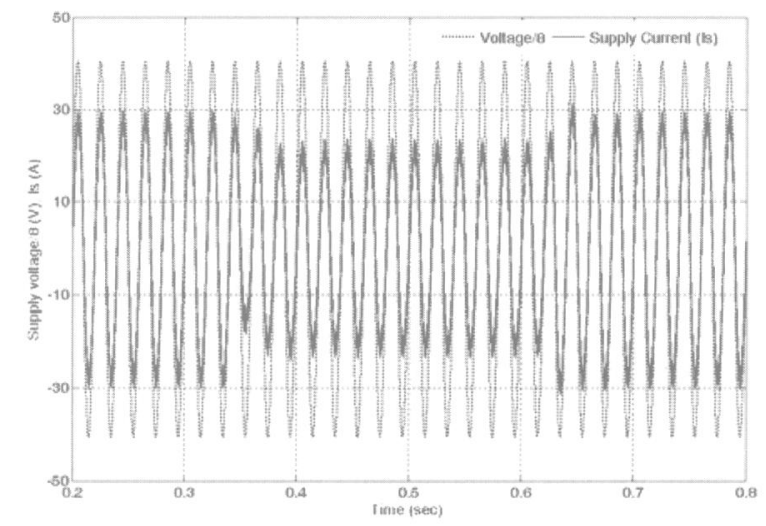

Figure 2 Supply voltage and supply current with wavelet controller based APF

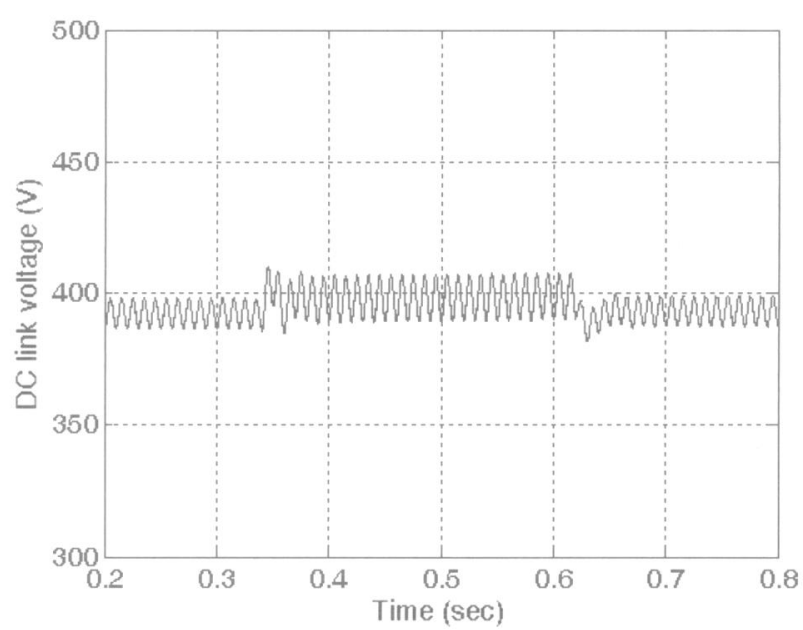

Figure 3 DC link voltage with wavelet controller

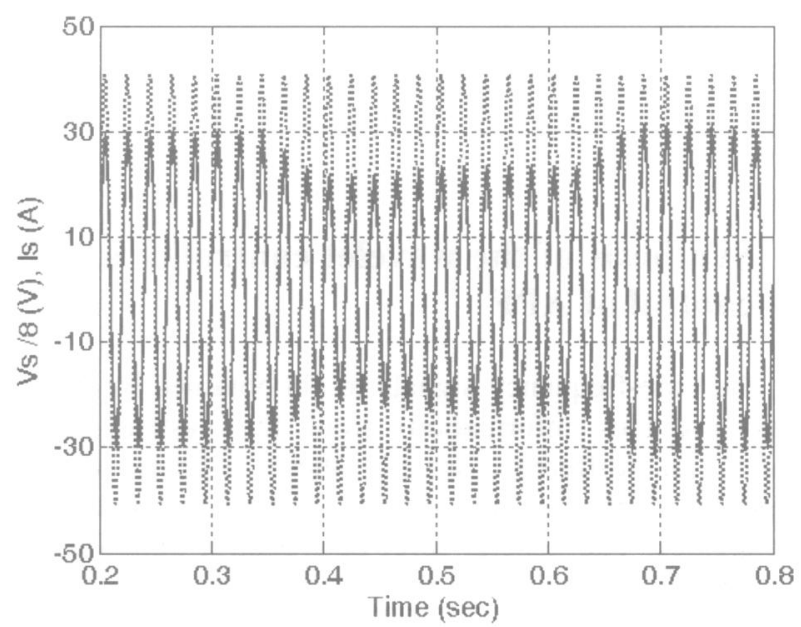

Figure 4 Supply voltage and supply current with PI controller based APF 


\section{References}

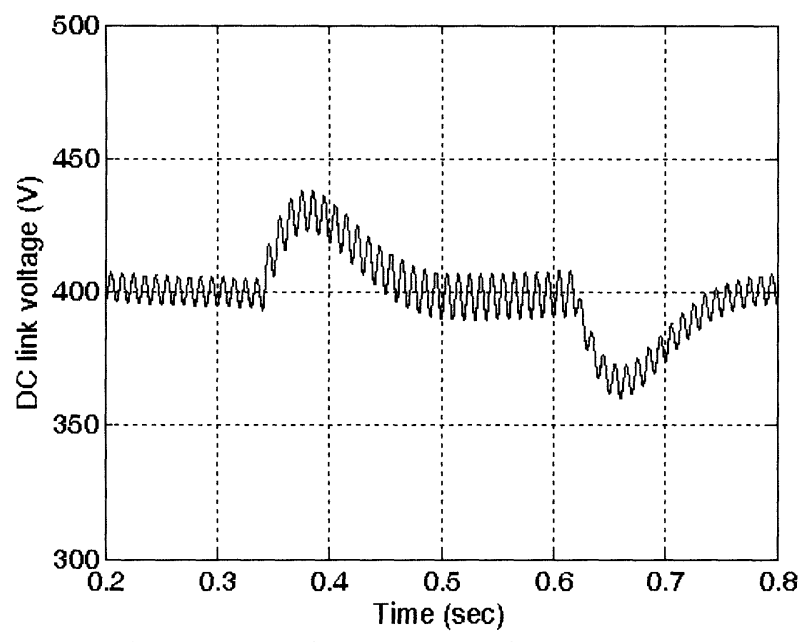

Figure 5 DC link voltage with PI controller

\section{Conclusions}

A wavelet-based controller has been proposed for shunt APF. The controller is based on wavelet analysis of the error and is a multi resolution extension of the conventional PI controller. The performance of the controller is satisfactory as compared to a conventional PI controller. From the example studies carried out it can be concluded that the controller is particularly efficient in controlling transient response effects. The better dynamic response is achieved as the wavelet controller is based on local frequency information. The novel technique of the generation of control signal ensures that even when the steady state error is zero, the controller can generate a steady control signal. The wavelet data window width is chosen optimally such that frequencies above $600 \mathrm{~Hz}$ are eliminated. This avoids the generation of high frequency spikes in the reference control current signal and ensures stability.
[1] S. Parvez and Z. Gao, "A wavelet based multiresolution PID controller", IEEE Trans. Ind. Appl., vol. 41, no. 2, March-Apr 2005, pp. 537-543.

[2] O. Poisson, P. Rioual, and M. Meunier, "Detection and measurement of power quality disturbances using wavelet transform", IEEE Trans. Power Delivery, vol. 15, no. 3, pp.1039-1044, July 2000.

[3] S. Santoso, P. Hoffman, "Power quality assessment via wavelet transform analysis", IEEE Trans. Power Delivery, vol. 11, no. 2, April 1996.

[4] M. Basu and B. Basu, "“'Application of Wavelet Transform to Power Quality (PQ) Disturbance Analysis", Proceedings of Power Electronics and Machine Drives, Edinburgh, vol. 1, pp. 269 -273, PEMD 2004.

[5] I. Daubechies, "The wavelet transform, time-frequency localization and signal analysis," IEEE Trans. Inf. Theory, vol. 36, no. 5, pp. 961-1005,Sep. 1990.

[6] S. G. Mallat, "A theory of multi-resolution signal decomposition: The wavelet representation," IEEE Trans. Pattern Anal. Mach. Intell., vol. 11, no. 7, pp. 674-693, Jul 1989.

[7] H. Akagi, "Trends in active power line conditioners," IEEE Trans. Power Electron., vol. 9, no. 3, pp. 263268, May 1994.

[8] T. Thomas, K. Haddad, G. Joos and A. Jaafari, "Design and performance of active power filters", IEEE Industry Appl. Mag., vol. 4, no. 5, pp. 38-46, Sept-Oct. 1998.

[9] K. Chatterjee, B. G. Fernandes, and G. K. Dubey “An instantaneous reactive volt-ampere compensator and harmonic suppressor system", IEEE Trans. Power Electronics, vol. 14, bo. 2, pp. 381-392, March 1999. 